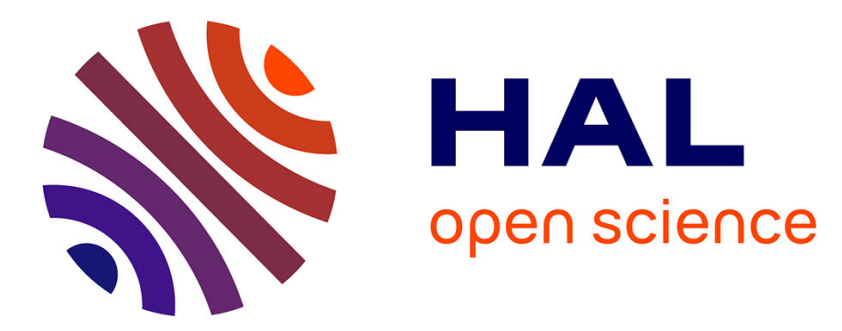

\title{
Beamforming Approach for Localization in WSN based Received Signal Strength
}

\author{
Maha Ben Zid, Kosai Raoof, Ammar Bouallegue
}

\section{To cite this version:}

Maha Ben Zid, Kosai Raoof, Ammar Bouallegue. Beamforming Approach for Localization in WSN based Received Signal Strength. TSP 2011 - 34th International Conference on Telecommunications and Signal Processing, Aug 2011, Budapest, Hungary. pp.n/a. hal-00690530

\section{HAL Id: hal-00690530 \\ https://hal.science/hal-00690530}

Submitted on 23 Apr 2012

HAL is a multi-disciplinary open access archive for the deposit and dissemination of scientific research documents, whether they are published or not. The documents may come from teaching and research institutions in France or abroad, or from public or private research centers.
L'archive ouverte pluridisciplinaire HAL, est destinée au dépôt et à la diffusion de documents scientifiques de niveau recherche, publiés ou non, émanant des établissements d'enseignement et de recherche français ou étrangers, des laboratoires publics ou privés. 


\title{
Beamforming Approach for Localization in WSN based Received Signal Strength
}

\author{
Maha Ben Zid, Kosai Raoof, and Ammar Bouallègue
}

\begin{abstract}
This paper is concerned with localization in wireless sensor networks (WSNs). We propose a novel localization algorithm based beamforming. The performed localization algorithm is adopted for a typical communication model in WSNs involving two clusters of sensors at both transmitter and receiver sides of the communication link. The set of collaborative sensor nodes located at the transmit cluster could be assimilated to a distributed Multiple Input Multiple Output (MIMO) system [1], [2], [3]. We tackle the task of estimating the position of randomly distributed sensor nodes and we present a novel algorithm based on the beamforming approach [4], [5] and the received signal strength $(\mathrm{SS})$ measurement. The correct operation of the proposed algorithm has been proved by simulation. The collected results for the sampling deviation of the estimator and the estimator variance are envisaged in order to evaluate the accuracy of the proposed algorithm.
\end{abstract}

Keywords-Beamforming, Collaborative sensors, Localization, Signal Strength, Wireless Sensor Networks.

\section{INTRODUCTION}

W IRELESS Sensor Network [6] is a significant emerging class of communication systems composed of large number of spatially distributed heterogeneous and single autonomous sensor nodes deployed in a sensing field. Sensor nodes are targeted to monitor a variety of ambient conditions such as environmental conditions. WSNs have attracted much attention in the last few years and are growing in maturity. The range of application areas of WSNs is large. WSNs are never limited to particular applications but are now applied in every day life. The various applications that could be addressed are not aware of localization task. Locating a sensor node involves the collection of location information from the radio signals traveling between a set of sensor nodes to a target sensor node. The location information consists of determining the spatial position of an object in the network by identifying its coordinates according to a reference coordinate system. Localization is a relevant processing to a vast number of scenarios. Some environmental application such as supervision of ambient conditions in forests require precise location for each sensor node since large scale fields could be deployed

Manuscript received May 20, 2011.

M. Ben Zid is with the University of Grenoble, GIPSA Laboratory, Department Image Signal, France and the National Engineering School of Tunis (ENIT), 6'COM Laboratory, Department of Technologies de l'Information et des Communications (TIC), Tunisia (e-mail: maha.benzid@gipsa-lab.grenoble-inp.fr).

K. Raoof is with the University of Grenoble, GIPSA-Lab, Department Image Signal, France. (e-mail: kosai.raoof@gipsa-lab.grenoble-inp.fr).

A. Bouallègue is with the National Engineering School of Tunis (ENIT), 6'COM Laboratory, Department of Technologies de l'Information et des Communications (TIC), Tunisia (e-mail: ammar.bouallegue@enit.rnu.tn). from a plane. Sensor nodes are deployed in the sensing field and exact location of some nodes remains uncertain. As such, an effective localization algorithm based on available information from some wireless sensor nodes is aimed to precise the location of some sensors. Another application is addressed in the case when a certain proportion of special sensing nodes can specify their own positions in the network. The positioning of a target node is identified based on the received signal strength from a set of sensor nodes placed at known locations. Thus, collecting the location information reveals an important activity for WSNs [7].

In this paper, we perform the beamforming approach for localization based on the received signal strength measurement. Position prediction which takes into consideration the signal strength measurement [8] has demonstrated efficient computation of the target node position by sampling several readings of the received signal strength and then evaluating the extremum over the sample readings.

The remainder of this paper proceeds as follows.

Section II presents the scenario adopted for the sensor node localization. Under the assumptions discussed in Section III, the paper gives in Section IV, the problem stating relative to the presented scenario. Section V provides the modeling of the proposed communication model. Additionally, the localization process based on beamforming approach and signal strength measurement is described in Section VI. The effectiveness of the proposed localization algorithm is proved by simulation results in Section VII. Finally, concluding remarks are summarized in Section VIII.

\section{SCENARIO DESCRIPTION}

The scenario to be considered in this paper is depicted in Fig. 1.

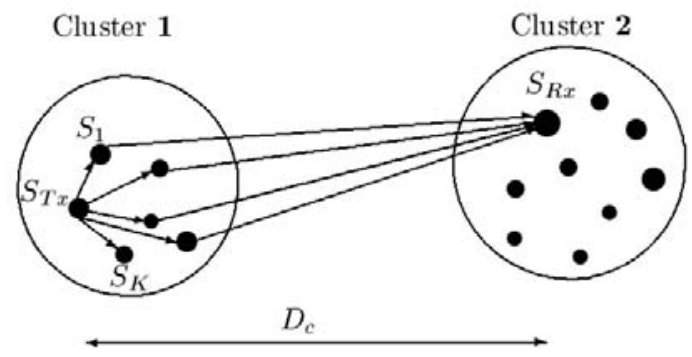

Fig. 1. Scenario 


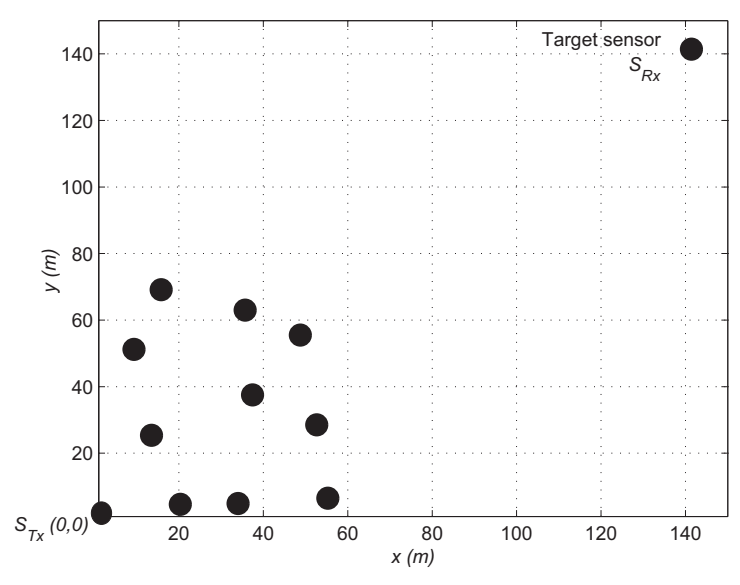

Fig. 2. Simulated scenario, $K=10$

We consider two clusters within wireless sensor network (WSN). Cluster 1 consists of a source sensor $S_{T x}$ and $K$ sensors $S_{1}, \ldots, S_{K}$ which are randomly distributed in a sensing area of $(70 \times 70) \mathrm{m}^{2}$. The source sensor sends redundant Binary Phase Shift Keying (BPSK) modulated data signal to the sensor nodes located in cluster 1 . The transmitting sensor nodes perform distributed MIMO system model. The set of the $K$ sensor nodes transmit in a collaborative manner to one target sensor node denoted $S_{R x}$. The target sensor could then transmit data to the sensors within cluster 2. Note that a $2 \mathrm{D}$ geometrical model is adopted. Sensors are distributed in the $(x-y)$ plane and source node is arbitrary placed at the origin of the system coordinate. The geometric spherical coordinates are defined by the triplet $(r, \theta, \varphi)$. Here, $\theta=\frac{\pi}{2}$. The simulated layout for the collaborative sensors with $K=10$ is depicted in Fig. 2.

\section{Assumptions}

The proposed localization algorithm is performed with respect to the following assumptions:

1) Assumption 1: We believe that WSNs is a class of distributed systems in which time synchronization between sensor nodes is one of the important goals that needs to be met. Time synchronization is a critical part of infrastructure in WSNs. In fact, sensor nodes require to maintain their local clocks in order to determine the events order [9]. As such, the use of synchronization protocols will allow processing different signals distributively improving the sensibility and the accuracy of the measurements in the network. Nevertheless, time synchronization is out of the scope of this paper. Time synchronization is not the goal of our work since available algorithms for synchronization exist in the literature. We consider that time synchronization between sensor nodes is assumed to be achieved. To examine in more details time synchronization in WSNs, please refer to [9].

2) Assumption 2: We consider that each sensor node is equipped with a single omnidirectional antenna. The choice for omnidirectional antenna (comparing with directional antenna) is motivated in this work since the devices that are deployed in sensor networks are small enough for evolutionary applications. As such, the choice of antenna type with minimum space requirement is of paramount importance for sensor network design.

\section{PRoblem STATEMENT}

The nearby communicating nodes in cluster 1 , each collecting their own data with identical messages transmit collaboratively to the same destination receiver sensor node $S_{R x}$. Assuming synchronization among the sensor nodes $S_{1}, \ldots, S_{K}$ and the transmission of identical messages, the beamforming scheme can be incorporated into the framework of wireless sensor networks [4]. The set of distributed sensor nodes forms a beam in the intended direction relative to the target sensor. $S_{R x}$ combines all the incoming signals from the $K$ distributed communicating nodes and the received signal strength is measured in order to estimate the location of the target sensor. Finally, an adequate algorithm for location estimation for the receive sensor node has to be performed. We propose a beamforming based localization algorithm. The target sensor node is assumed to be at approximative distance, denoted $D_{c}$ far away from the transmit sensor node $S_{T x}$. The detection of the target node relies on determining the steering vector that fits the location of the target sensor node $S_{R x}$.

\section{SYSTEM MODELING}

Each propagation link between sensor $S_{k} ; k=1, \ldots, K$ and the target sensor $S_{R x}$ is characterized by :

1) A Rayleigh distributed attenuation $\lambda_{k} ; k=1, \ldots, K$

2) A delay $\tau_{k}$ relative to the reference sensor $S_{T x}$ expressed as:

$$
\tau_{k}=d_{k} \cdot \frac{\cos \left(\alpha_{k}\right)}{c} \quad ; k=1, \ldots, K
$$

- $d_{k}$ : Distance between sensor $S_{k}$ and the source node $S_{T x} ; k=1, \ldots, K$

- $\alpha_{k}$ : Direction of arrival relative to the target sensor $S_{R x} ; k=1, \ldots, K$

- $c$ : Speed of the light

3) A dephasing $\psi_{k}$ :

$$
\psi_{k}=2 \pi \cdot f_{c} \cdot \tau_{k} \quad ; k=1, \ldots, K
$$

- $f_{c}$ : The carrier frequency

The channel gain relative to the transmission link between sensor node $S_{k}$ and the receive sensor node $S_{R x}$ is then:

$$
h_{k}=\lambda_{k} \cdot e^{j \psi_{k}} \quad ; k=1, \ldots, K
$$

A Hadamard Direct-Sequence Code Division Multiple Access (DS-CDMA) code is designed for redundant transmitted BPSK data spreading. Walsh-Hadamard codes [10] are perfectly orthogonal and employed to avoid interference among users in the propagation link. These codes are exploited for sensor identification and help to mitigate noise effect. The codes for users could be expressed as the columns (or 
the rows) of the Walsh-Hadamard matrix $C$. The simplest Hadamard matrix codes are :

$$
C_{1}=1 \quad \text { and } \quad C_{2}=\left[\begin{array}{cc}
1 & 1 \\
1 & -1
\end{array}\right]
$$

This can be extended to a generic matrix notation using a recursive technique. In fact, if $C$ is a Hadamard matrix of order $l$ (the spreading code length), then becomes the $2 l$ order Hadamard matrix :

$$
\left[\begin{array}{cc}
C & C \\
C & -C
\end{array}\right]
$$

and

$$
C_{2^{l}}=C_{2} \otimes C_{2^{l-1}}
$$

$\otimes$ denotes the Kronecker product.

$C_{2^{l-1}}, 2 \leq l$ is expressed as :

$$
C_{2^{l}}=\left[\begin{array}{cc}
C_{2^{l-1}} & C_{2^{l-1}} \\
C_{2^{l-1}} & -C_{2^{l-1}}
\end{array}\right]
$$

Given a sensor $S_{k}$ with cartesian coordinates $\left(x_{k}, y_{k}, z_{k}=\right.$ $0)$, the associate spherical coordinates are $\left(r_{k}, \frac{\pi}{2}, \varphi_{k}\right)$. where:

- $r_{k}=\sqrt{x_{k}^{2}+y_{k}^{2}} \quad ; k=1, \ldots, K$

- $\varphi_{k}=\tan ^{-1}\left(\frac{y_{k}}{x_{k}}\right) \quad ; k=1, \ldots, K$

Let $\beta=\frac{2 \pi}{\lambda}$. The steering vector is expressed as :

$$
V s=\left[e^{\left(-j \beta R_{1}\right)}, \ldots, e^{\left(-j \beta R_{K}\right)}\right]
$$
here:

$\left(D_{c}, \frac{\pi}{2}, \varphi_{c}\right)$ are the target node spherical coordinates.

- $D_{c}$ : Distance between source node $S_{T x}$ and the target sensor $S_{R x}$

- Distance $R_{k} ; k=1, \ldots, K$ is the Euclidean distance ${ }^{1}$ between the target sensor $S_{R x}$ and sensor $S_{k}$ which is given by:

$$
R_{k}=\left|D_{c}-d_{k}\right| \quad ; k=1, \ldots, K
$$

Euclidean distance $R_{k}$ can be approximately expressed as [11]:

$$
\begin{aligned}
R_{k} & =\sqrt{D_{c}^{2}+d_{k}^{2}-2 d_{k} D_{c} \cos \left(\varphi_{c}-\varphi_{k}\right)} \\
& \simeq D_{c}-d_{k} \cos \left(\varphi_{c}-\varphi_{k}\right) \\
& =D_{c}-d_{k} \cos \left(\zeta_{k}\right)
\end{aligned}
$$

where:

- $\zeta_{k}=\left(\varphi_{c}-\varphi_{k}\right) ; k=1, \ldots, K$ is the direction of transmitted signal vector relative to sensor $S_{k}$.

- $d_{k c} ; k=1, \ldots, K$ (refer to Fig. 3) denotes the distance between sensor $S_{k}$ and the target node $S_{R x}$.

${ }^{1}$ The Euclidean distance between two points $\mathrm{A}$ and $\mathrm{B}$ with respective coordinates $A\left(x_{A}, y_{A}, 0\right)$ and $B\left(x_{B}, y_{B}, 0\right)$ is:

$$
d_{\text {euc }}=\sqrt{\left(x_{A}-x_{B}\right)^{2}+\left(y_{A}-y_{B}\right)^{2}}
$$

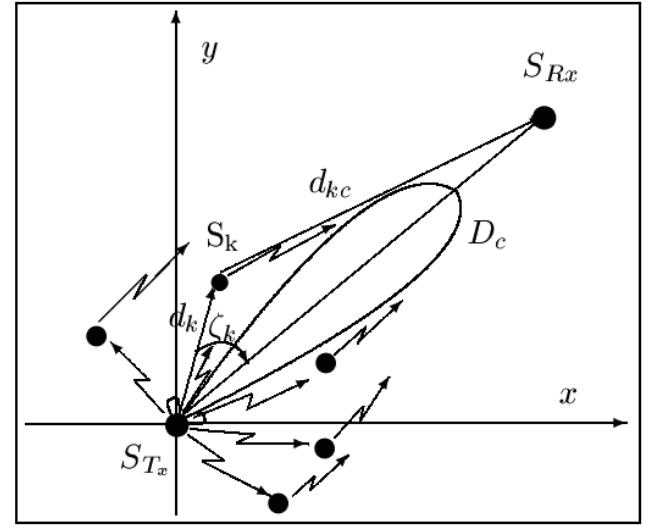

Fig. 3. Collaborative beamforming based localization

\section{LOCALIZATION PROCESS BASED ON SIGNAL STRENGTH MEASUREMENT}

The target object is said to be detected if the azimuthal coordinate $\varphi_{c}$ is accurately estimated. Antennas of the whole sensors $S_{1}, \ldots, S_{K}$ are steered in different directions relative to a possible location of the target sensor. This assumes a stepscanning angle. As such, the steering vector is expressed in function of the angle $\varphi_{c}$.

The proposed algorithm for sensor localization is described as below:

1) Start by finding the position of the $K$ sensors within cluster 1.

2) Sensors $S_{1}, \ldots, S_{K}$ collaborate to form a common message so that to steer a beam in the direction of $S_{R x}$ (See Fig. 3). This process is repeated for all possible values of the azimuthal angle $\varphi_{c}$ according to a specific scanning angle step.

3) $S_{R x}$ feedbacks the CDMA code to the sensors $S_{1}, \ldots, S_{K}$ within cluster 1 . We assume that the propagation channel is symmetric.

4) The received data at sensors $S_{1}, \ldots, S_{K}$ is despreaded and broadcasted to the sensor node $S_{T x}$.

5) The received signal strength is measured for all possible angles relative to the target node location.

6) The measurement of the overall received signal strength is performed for 1000 iterations of the Monte Carlo computational algorithm. The estimated angle of the target sensor corresponds to the maximum signal strength value measured at each iteration.

The accuracy of the proposed algorithm is evaluated in terms of statistical parameters under different signal-to-noise ratio levels. The simulated system is sketched in Fig. 4.

We adopt a filtering scheme matched to the spreading code. The most interesting property of the matched filter is that when a signal is corrupted with an additive white gaussien noise (AWGN), the filter with the impulse response matched to this signal maximizes the output signal-to-noise ratio (SNR) [12]. The channel matched filter performed in this work is based on the square root cosine filter. 


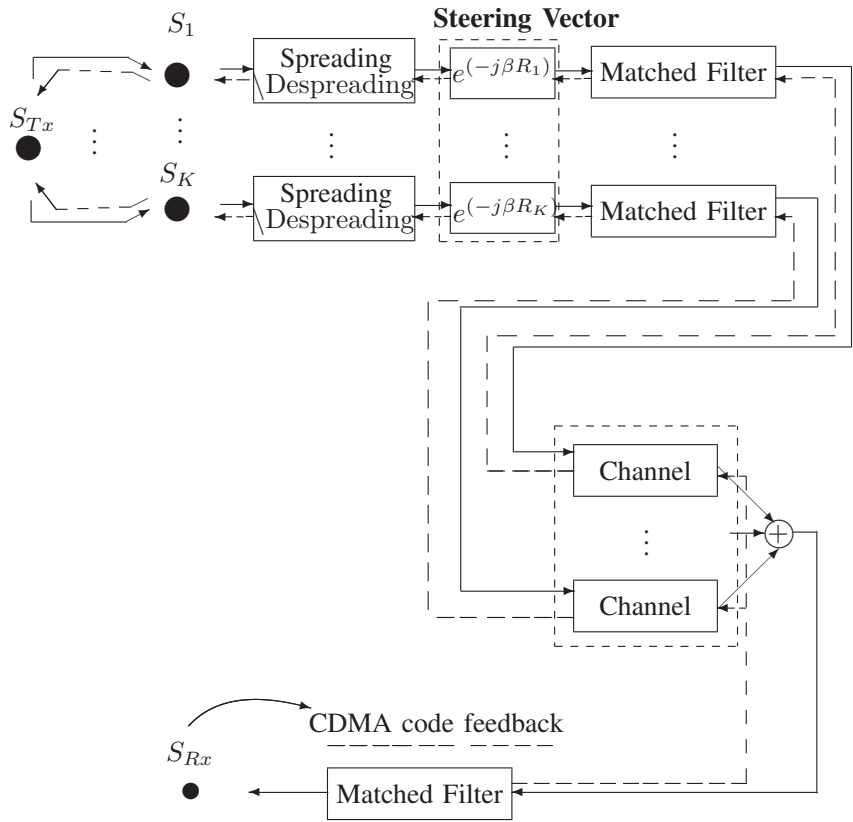

Fig. 4. The location estimation process

\begin{tabular}{|l|l|}
\hline Simulation parameter & Value \\
\hline \hline Spreading factor & 16 \\
\hline Distance $\left(D_{c}\right)$ & $200 \mathrm{~m}$ \\
\hline Number of scanning angles & 50 \\
\hline Oversampling factor for Nyquist filter & 8 \\
\hline
\end{tabular}

TABLE I

MAIN SIMULATION PARAMETERS

\section{Simulation RESUlTS AND PERFORMANCE EVALUATION}

We present in this section, the simulation results in terms of statistical parameters. We firstly, provide the main simulation parameters which are provided by the following table:

We adopt the histogram method for determining the probability density function (pdf) of the estimated azimuth angle relative to the target sensor node. The cumulative histogram counts the number of observations that fall into each of the disjoint ranges of angles. Fig. 5 shows that the pdf of the estimated angle obtained beyond 1000 runs at a signal-to-noise ratio of $7 \mathrm{~dB}$. approaches the gaussian distribution.

We evaluate based on the Monte Carlo simulation, the performance of the developed algorithm for location estimation. We denote in the following, the estimator for the azimuthal angle by $\hat{\varphi}_{c}$. We derive the statistical metrics for the estimator involving both the average sampling deviation and the variance of the estimator. The characterization of the estimator will be addressed for different numbers of the collaborative sensors.

\section{A. Sampling deviation of the estimator}

The sampling deviation of the estimator denoted by $d$ is expressed for a given sample $s$ by equation (11).

$$
d(s)=\left[\hat{\varphi}_{c}(s)-\mathrm{E}\left(\hat{\varphi}_{\mathrm{c}}\right)\right]
$$

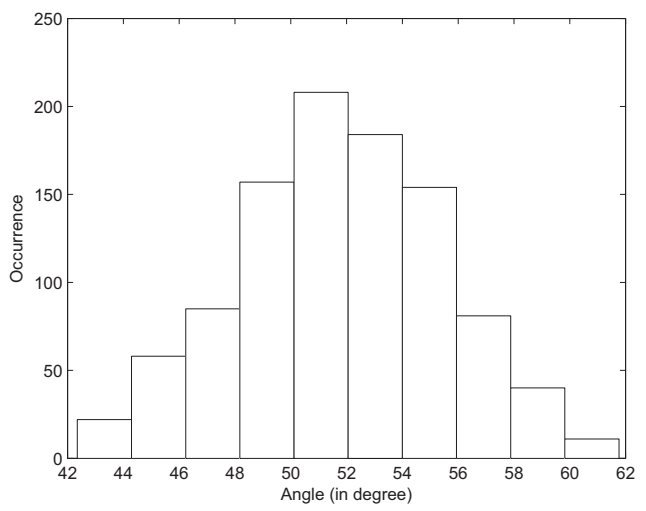

Fig. 5. Histogram for 1000 runs and $S N R=7 d B$

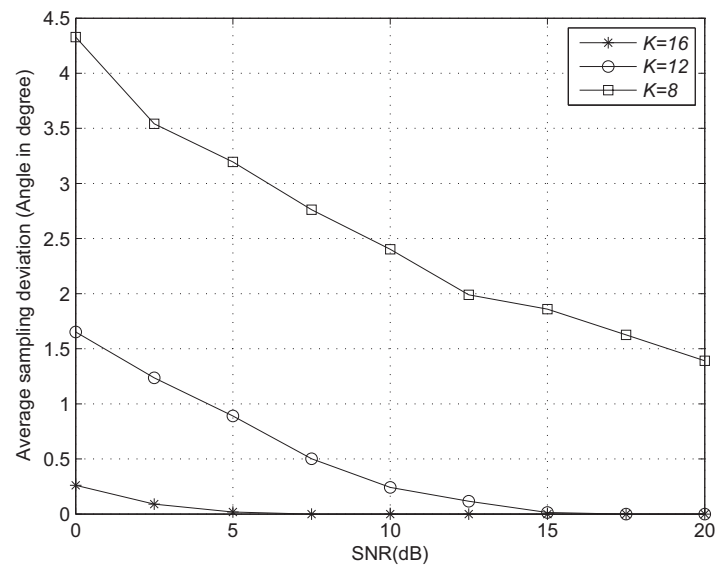

Fig. 6. Average sampling deviation as a function of received SNR and variable number of collaborative sensors

where:

- $\mathrm{E}($.$) is the expectation operator.$

- $\mathrm{E}\left(\hat{\varphi}_{\mathrm{c}}\right)$ evaluates the sample average of the estimated angle.

The average over absolute values of the sampling deviation associated to the estimator helps to evaluate the error of the estimator.

Plotted curves for the average sampling deviation are shown in Fig. 6. The obtained curves are presented as a function of the received signal-to-noise ratio at the target sensor for variable number of the collaborative sensor nodes.

The simulation results for the average sampling deviation over the received signal-to-noise ratio as presented in Fig. 6 show that deploying 16 communication sensors leads to more accurate localization process. Using 12 sensor nodes remains satisfying. Nevertheless, when cluster 1 only consists of 8 sensors, the location variance is tolerable at only high signalto-noise ratio levels.

\section{B. Variance of the estimator}

The variance of an estimator measures the deviation of the estimated location target node from its mean value. It is used 


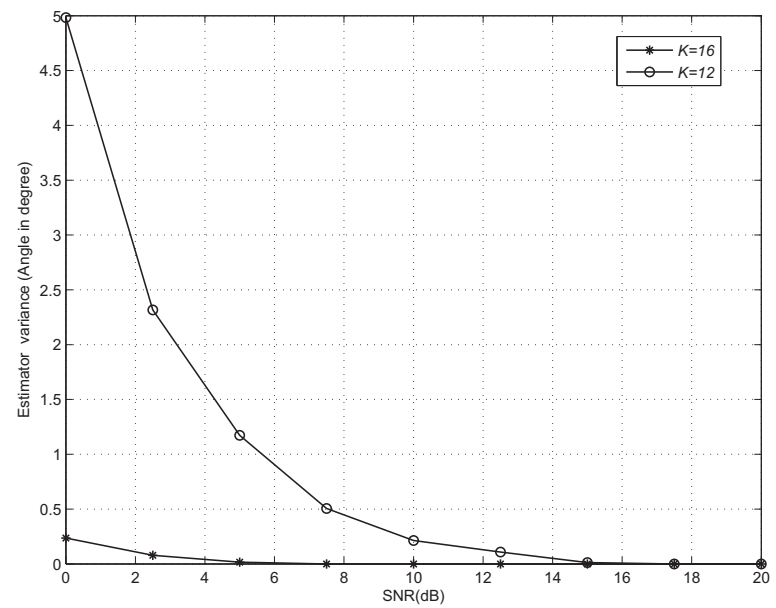

Fig. 7. Estimator variance as a function of received SNR and variable number of collaborative sensors

to quantify the difference between an estimated value and the true value of the quantity being estimated. The variance of the estimator could be then expressed as:

$$
\operatorname{var}\left(\hat{\varphi}_{c}\right)=\mathrm{E}\left[\left(\hat{\varphi}_{\mathrm{c}}-\mathrm{E}\left(\hat{\varphi}_{\mathrm{c}}\right)\right)^{2}\right]
$$

Simulation results for the estimator variance with variable number of collaborative sensors are presented in Fig. 7. The obtained results show that the target sensor location is better estimated if more collaborative sensors are used. When 16 collaborative sensors are introduced, the variance of the estimator is almost negligible even for low signal-to-noise ratio and the location estimation is shown to be more accurate then deploying 12 sensor nodes.

\section{CONCLUSION}

This paper presents the analysis for an adequate localization technique for wireless sensor network with collaborative sensors performing a beamforming approach. The localization technique is carried out by measuring the received signal strength for all possible physical locations of the target sensor. Monte Carlo simulation is performed and the estimation of the receive sensor's azimuth angle is computed as the sample mean of the estimated angles derived from the peak values of the received signal strength. The collaborative wireless sensors physically distributed at the same cluster collaborate to steer the beam on the direction of the target node. Statistical parameters are used to evaluate the accuracy of the proposed localization algorithm and illustrative numerical examples are presented. The simulation results have demonstrated that the proposed scheme performs well for localization in WSNs. It is shown that the localization method provides better precision when more sensor nodes jointly communicate in the network. As we have proved the correctness of the novel algorithm for localization in WSNs for a typical communication model, this work could be improved by studying the impact of imperfections in time synchronization on the accuracy of the proposed localization algorithm.

\section{REFERENCES}

[1] K. Raoof, M. Ben Zid, N. Prayongpun, and A. Bouallègue. (2011, April). Advanced MIMO techniques: Polarization diversity and antenna selection [Online]. pp. 3-56. Available:http://www.intechopen.com/articles/show/title/advancedmimo-techniques-polarization-diversity-and-antenna-selection

[2] M. Ben Zid, K. Raoof, and A. Bouallègue,"MIMO Systems and Cooperative Networks Performance," in Cognitive radio,Ed. USA: Scientific Research Publishing, to be published.

[3] K. Raoof and H. Zhou, Advanced MIMO Systems. USA: Scientific Research Publishing, 2009.

[4] H. Ochiai, H. Imai, "Collaborative Beamforming," in New Directions in Wireless Communications Research, 1st ed., V. Tarokh, Ed. Springer, 2009.

[5] B. D. Van Veen, K. M. Buckley, "Beamforming: A Versatile Approach to Spatial Filtring," IEEE ASSP Magazine, vol. 5, no. 2, pp. 4-24, 1988.

[6] F. I. Akyildiz, W. Su, Y. Sankarasubramaniam, E. Cayirci, "A Survey on Sensor Networks," IEEE Comm. Magazine, vol. 40, no. 8, pp. 102-114, 2002.

[7] S. Pandey, P. Agrawal, "Survey of Localization Techniques in Wireless Networks," Journal of the Chinese Institute of Engineers, vol. 29, no. 7, pp. 1125-1148, 2006.

[8] C. Papamanthou, F. P. Preparata, R. Tamassia, "Algorithms for Location Estimation Based on RSSI Sampling," in Lecture Notes in Computer Science, vol. 5389/2008, S. P. Fekete, Ed. Berlin, Heidelberg: Springer Verlag, 2008, pp. 72-86.

[9] X. Liu, S. Zhou, "Evaluation of several time synchronization protocols in WSN" in Proc. International Conference of Information Science and Management Engineering, Xi'an, 2010, pp. 488-491.

[10] T. A. Wysocki, "Modified Walsh-Hadamard Sequences for DS-CDMA Wireless systems," International Journal of Adapt. Control and Signal Processing, vol. 16, pp. 589-602, 2002.

[11] M. F. A. Ahmed, S. Vorobyov, "Collaborative Beamforming for Wireless Sensor Networks with Gaussian Distributed Sensor Nodes," IEEE Trans. on Wireless Communications, vol. 8, no. 2, pp. 638-643, 2009.

[12] J. G. Proakis, "Digital Communications," 3rd ed, Ed. New York: Mac Graw-Hill Higher Education, 2000. 